\title{
Cuatro nuevos registros de especies del género Elaphoglossum (Dryopteridaceae) para el Perú
}

\section{Three new records of species of genera Elaphoglossum (Dryopteridaceae) to Peru}

\author{
L. Franco Mellado, Joaquina Alban y Blanca León
}

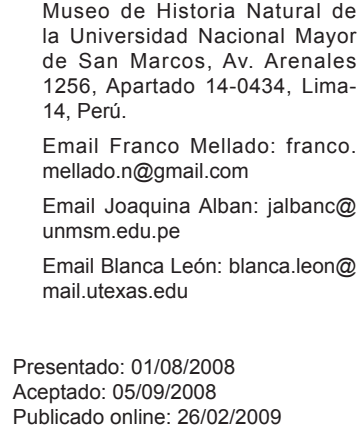

Museo de Historia Natural de la Universidad Nacional Mayor de San Marcos, Av. Arenales 1256, Apartado 14-0434, Lima1256, Ap

Email Franco Mellado: franco. mellado.n@gmail.com

Email Joaquina Alban: jalbanc@ unmsm.edu.pe

Email Blanca León: blanca.leon@ mail.utexas.edu

Presentado: 01/08/2008

Aceptado: 05/09/2008

Publicado online: 26/02/2009

\begin{abstract}
Resumen
Se confirma y registra para la flora peruana a las especies Elaphoglossum lloense, E. murinum, E. neei y E. puberulentum, provenientes del Parque Nacional Yanachaga-Chemillén, Oxapampa, Pasco. Con estos registros se incrementa a 142 el número de taxones conocidos en el género para el Perú.
\end{abstract}

Palabras claves: Dryopteridaceae, Elaphoglossum, pteridófitas, helechos, Perú

\section{Abstract}

Elaphoglossum lloense, E. murinum, E. neei and E. puberulentum are confirmed and reported for the Peruvian flora, all of which were found in Yanachaga-Chemillén National Park, Oxapampa,Pasco These new records increases to 142 the total number of taxa known in the genus for Peru.

Keywords: Dryopteridaceae, Elaphoglossum, pteridophytes, ferns, Peru

\section{Introducción}

En el Perú el género Elaphoglossum era conocido con 138 especies (Mickel 1991). La continua exploración botánica del país y el estudio de este género ha resultado en nuevos e interesantes registros (Rojas-Alvarado 2002; Mellado \& León 2007). El mayor número de taxones en el género se distribuyen en las vertientes orientales de la cordillera de los Andes (León 2007), alcanzando su mayor diversidad en el Parque Nacional Yanachaga-Chemillén entre los 1700 a $2700 \mathrm{~m}$ de altitud (Mellado 2007).

Los nuevos registros del género Elaphoglossum para el Perú que se presentan en este artículo son parte de los resultados del proyecto de investigación de Mellado (2007). La ubicación taxonómica del género ha tenido varios cambios, aquí se sigue la clasificación de Smith et al. (2006).

Tres de las especies que se presentan en este trabajo (Elaphoglossum murinum, E. neei y E. puberulentum) han sido descritas inicialmente para Bolivia (Kessler \& Mickel 2006). Mientras que de Elaphoglossum lloense se conocen registros en Bolivia, Colombia y Ecuador.

Se incluye además información adicional para Elaphoglossum puberulentum, la cual era conocida previamente sólo de material estéril. Para las otras especies se presentan nuevos rangos de variaciones morfológicas producto de la medición de muestras recolectadas en diferentes puntos de muestreo y observaciones para distinguirlas de otras especies afines presentes en la flora peruana.

\section{Materiales y métodos}

El material estudiado se ha depositado preferentemente en los Herbarios San Marcos (USM), Missouri Botanical Garden (MO) y la colección del Jardín Botánico de Missouri: Herbario Selva Central-HOXA ubicada en la ciudad de Oxapampa.

\section{Resultados}

Como resultado de un estudio minucioso de campo, en el Parque Nacional Yanachaga-Chemillén se han registrado tres especies, E. murinum, E. neei y E. puberulentum, descritas previamente para Bolivia (Kessler \& Mickel 2006) y una especie,
E. lloense, descrita para Ecuador (Jørgensen \& León Yánez 1999) y considerada restringida a ese país (Navarrete 2000); de esta manera se incrementa el número de taxones conocidos del género Elaphoglossum en el Perú de 138 a 142.

\section{Elaphoglossum Iloense (Hook.) T. Moore Index Filic. 11. 1857.}

\section{Acrostichum lloense Hook.}

Tipo: ECUADOR, Jameson s.n., Pichincha (holótipo: K).

Rizoma largamente repente, $1 \mathrm{~mm}$ de diámetro; escamas del rizoma linear-lanceoladas, marrón-castańas, enteras, 3-3,5 x 0,5-0,8 $\mathrm{mm}$; filopodio ausente; hojas $8-20 \mathrm{~mm}$ distanciadas, 7-20 x 1-2 cm; pecíolo 1/2-2/3 del largo de la hoja estéril; escamas del pecíolo ovado-lanceoladas, enteras a dentadas, 2-3 x 0,8-1 mm, castańas, esparcidas; lámina lanceolada a angostamente elíptica, cartácea, ápice de agudo a acuminado, raramente obtuso, base cuneada, borde entero; nervaduras conspicuas, 1 $\mathrm{mm}$ espaciadas, en un ángulo de $60^{\circ}$ con respecto a la costa, libres; hidatodos ausentes; escamas de la lámina lanceoladas, enteras a ocasionalmente dentadas, $1-2 \times 0,2-0,3 \mathrm{~mm}$, marrones a marrón anaranjada, lustrosas, hastadas; escamas de la costa deciduas; escamas de la haz iguales a las del envés; hoja fértil más larga o igual del largo de la hoja estéril; pecíolo 1/5-1/6 de la longitud de la hoja fértil; lámina fértil lanceolada, ápice agudo a obtuso, base cuneada, borde entero; escamas interesporangiales lanceoladas, enteras a dentadas, marrones, ligeramente lustrosas, $2 \times 1 \mathrm{~mm}$, hastadas, abundantes, escamas de la costa ausentes.

Hierba, escandente; (2400-) 2657-3419 msnm. En bosque montano húmedo hasta los fragmentos de bosque en pajonales.

Observaciones: Elaphoglossum. lloense (Sección Squamipes, sub sección squamipedia) está relacionada a E. squamipes (Hook.) T. Moore, sin embargo morfológicamente difiere de este por presentar el ápice de la lámina de agudo a acuminado y las escamas de las láminas generalmente enteras y hastadas, mientras que $E$. squamipes presenta el ápice obtuso y las escamas de la lámina por lo general son dentadas. 
Material examinado: PERÚ: Dep. Pasco. Prov. Oxapampa. Lanturachi: Zona de Amortiguamiento del P.N. Yanachaga Chemillén, sector Santa Bárbara, Milpo, 2900 m, 01-feb-2005, L. F. Mellado, A. Monteagudo \& R. Francis J., 2767 (USM, MO, HOXA);(USM, MO, HOXA, HUT, AMAZ, MOL); Oxapampa: Sector Quebrada San Alberto, P. N. Yanachaga-Chemillén, 2657 m, 24-oct-2004, L. F. Mellado \& E. Becerra, 2062 (USM, MO, HOXA); 2658 m, 24-oct-2004, L. F. Mellado \& E. Becerra, 2071 (USM, MO, HOXA).

\section{Elaphoglossum murinum M. Kessler \& Mickel, Brittonia 58(2): 109. 2006}

Tipo: BOLIVIA. La Paz: Prov. Inquisivi, comunidad Choquetanga-Cuchiwasi, bajando Pabellonani a $7 \mathrm{~km}$ al NE de Choquetanga, $14^{\circ} 48^{\prime} \mathrm{S}, 67^{\circ} 17^{\prime} \mathrm{W}, 3330 \mathrm{~m}, 19$ ene 1994 , Salinas 2199 (holótipo: NY; isótipo: LPB).

Rizoma largamente repente, ramificado, 2-2,5 $\mathrm{mm}$ de diámetro; escamas del rizoma lineares, marrón negro-rojizas, lustrosas, dentadas, 3-4 x 0,2 mm, ligeramente crispadas; filopodio presente, $7-8 \mathrm{~cm}$ de largo, algunas veces cubierto por escamas; hojas 5-15 mm distanciadas, $35-39 \times 2,2-2,5 \mathrm{~cm}$; pecíolo $1 / 2$ a ca. 1/3 del largo de la hoja estéril; escamas del pecíolo lineares, enteras, 2 × 0,2 mm, marrón negro-rojizas, acompańadas por escamas más pequeńas adpresas y por puntos de resina; lámina elípticas a ligeramente oblongas, sub-cartácea, ápice largamente cuspidado, base cuneada raro redondeada, borde entero, lámina verde olivo; nervaduras conspicuas, $1 \mathrm{~mm}$ espaciadas, en un ángulo de $60-70^{\circ}$ con respecto a la costa, libres; hidatodos ausentes; escamas de la lámina lanceoladas, enteras, 1-1,5 x $0,2 \mathrm{~mm}$, más evidentes en el margen de la hoja, las escamas de la lámina impregnadas de resina, lámina con puntos de resina; escamas de la costa lanceoladas, enteras, marrón rojizas a marrón negro-rojizas, 1,5 x 0,2 mm; escamas de la haz iguales a las del envés; hoja fértil más angosta y ligeramente más corta del largo de la hoja estéril; pecíolo ca. 1/6 de la longitud de la hoja fértil; lámina fértil elíptica, ápice ausente, base cuneada; escamas interesporangiales ausentes; costa con escamas lanceoladas, enteras, marrón rojizas, $1 \mathrm{~mm}$ de largo, impregnadas de resina.

Hierba, epífita, péndula; 2891-3419 m de altitud. En bosque montano y en fragmentos de bosque en puna.

Observaciones. Según la subdivisión infra genérica de Mickel \& Atehortúa (1980), E. murinum pertenecería a la sección lepidoglossa y a la subsección Petiolosa (Kessler \& Mickel 2006; Mellado 2007), estando relacionada por lo tanto con las especies que presentan puntos de resina sobre la lámina, y dentro estas se relaciona mejor con E. petiolosum (Desv.) T. Moore. Sin embargo, E. murinum se diferencia por presentar el rizoma largamente repente, ramificado con escamas lineares (3-4 x 0,2 mm,) dentadas y ligeramente crispadas, mientras que E. petiolosum presenta un rizoma cortamente repente con escamas lineares, enteras o con dientes esparcidos, 4-6 x 0,5 mm longitud .

Material examinado: PERÚ: Dep. Pasco. Prov. Oxapampa. Lanturachi: P.N. Yanachaga Chemillén, Sector Santa Bárbara, Camino de Milpo a Cueva Blanca, colecta al lado del camino., 2648-2891 m, 18-mar-2004, L. Franco Mellado N., 1474 (USM, MO, HOXA); Lanturachi: Zona de Amortiguamiento del P.N. Yanachaga Chemillén, sector Santa Bárbara, Santa Bár- bara, 3389-3419 m, 29-ene-2005, L. F. Mellado \& E. Ortiz V., 2691 (USM, MO, HOXA).

\section{Elaphoglossum neei M. Kessler \& Mickel, Brittonia 58(2): 111. 2006}

Tipo: BOLIVIA. La Paz: Prov. Murillo, $1 \mathrm{~km} \mathrm{~W}$ of Estancia Islani, 1605'S, 68²03'W, 1700-2050 m, 18 Jun 1998, Nee \& Bohs 49764 (holótipo: NY; isótipos: LPB, MO, USZ, UT).

Rizoma cortamente repente, 3-5 mm de diámetro; escamas del rizoma lineares, marrón-oscuro-rojizas, ciliadas o con dientes piliformes, 3-4 x 0,5-0,7 mm, lustrosas; filopodio presente, 9-14 cm de largo; hojas fasciculadas, 34-52 x 1.5-3.3 cm; pecíolo 1/5-1/3 del largo de la hoja estéril; escamas del pecíolo linearlanceoladas, con dientes largamente piliformes, $2-4$ x $0,7-0,8$ $\mathrm{mm}$, castaño anaranjadas con el borde y los dientes ligeramente más oscuros, esparcidas; lámina angostamente elíptica, cartácea a sub-cartácea, ápice acuminado a largamente cuspidado, base angostamente cuneada a ligeramente decurrente, borde irregular y ligeramente crenado, lámina marrón-anaranjada, la haz más oscura; nervaduras conspicuas, $1 \mathrm{~mm}$ espaciadas, en un ángulo de $60-70^{\circ}$ con respecto a la costa, libres; hidatodos ausentes; escamas de la lámina lanceoladas a ovadas-lanceoladas, con largos dientes piliformes, 0,5-1 x 0,5-0,6 mm, marrón-anaranjadas, conspicuamente dispuestas sobre las venas secundarias, deciduas con el tiempo dejando una punto oscuro, las escamas del borde de la lámina llegando hasta 1,5 mm de largo; escamas de la costa lineares, ciliadas con dientes piliformes, marrón-anaranjadas, 2 x 0,6 mm, algunas veces el ápice y/o la base de la escama más oscuras, mixtas con escamas iguales a la de la lámina, algunos puntos de resina esparcidos en la lámina; escamas de la haz lanceoladas, ligeramente ciliadas, blanquecino anaranjadas, $0,5-1 \times 0,2 \mathrm{~mm}$, algunas veces reducidas a tricomas estrellados, dispuestas sobre las venas secundarias, aun que menos abundantes que las del envés; hoja fértil igual a ligeramente más corta del largo de la hoja estéril; pecíolo ca. 1/2 de la longitud de la hoja fértil; lámina fértil linear, más angosta que la hoja estéril, 0,9-1,1 $(1,7) \mathrm{cm}$ de ancho, ápice acuminado a cortamente cuspidado, base truncada a redondeada, borde de entero a conspicuamente crenado; escamas interesporangiales deciduas, costa con escamas linear-lanceoladas, con largos dientes piliformes, 2 x 0,5-0,6 mm, castańo anaranjadas, ascendentes; haz con abundantes escamas de $1 \mathrm{~mm}$ de largo, linear-lanceoladas, con largos dientes piliformes, castaño anaranjadas, las de la costa más largas $(1,5 \mathrm{~mm})$.

Hierba, epífita facultativa, cuando epífita péndula, formando matas; 2400-3419 msnm. En bosque montano húmedo hasta los fragmentos de bosque en puna.

Observaciones: Esta especie se encuentra ubicada en la Sección Lepidoglossa y en la subsección Polytrichia. Elaphoglossum neei se encuentra relacionada a E. rimbachii (Sodiro) C. Chr., sin embargo se diferencia de esta, por presentar las escamas del envés de la lámina más esparcidas (dejando ver la lámina) y ubicadas sobre las venas secundarias, mientras que en E. rimbachii las escamas cubren completamente en el envés de la lámina.

Material examinado: Perú. Dep. Pasco. Prov. Oxapampa. Huancabamba: P. N. Yanachaga Chemillén, Sector Quebrada Yanachaga., 2660 m, 13-oct-2004, L. F. Mellado \& E. Becerra, 1911 (USM, MO, HOXA); 1912 (USM, MO, HOXA); 2410 
m, 20-set-2004, L. F. Mellado \& E. Becerra, 1863 (USM); 2545 m, 22 set 2004, L. F. Mellado \& E. Becerra, 1891 (USM); 2900 m, 01 feb 2005, L. Franco Mellado N., A. Monteagudo \& R. Francis J., 2771 (USM); Lanturachi: P.N. Yanachaga Chemillén, Sector Santa Bárbara, Milpo, 2891 m, 16 mar 2004, L. F. Mellado \& A. Monteagudo M., 1459 (USM, MO, HOXA); sector Santa Bárbara, Santa Bárbara, 3379 m, 27 ene 2005, L. F. Mellado \& E. Ortiz V., 2639 (USM, MO, HOXA, HUT); 2657 (USM, MO, HOXA); 3389-3419 m, 29 ene 2005, L. F. Mellado \& E. Ortiz V., 2695 (USM, MO); Oxapampa: Sector Quebrada San Alberto, P.N. Yanachaga-Chemillén., 2400 m, 21 ago 2004, L. F. Mellado \& Rolando Francis J., 1568 (USM); 2820 m, 22 Octubre 2004, L. F. Mellado \& E. Becerra, 2027 (USM, MO); 2658 m, 24 Octubre 2004, L. F. Mellado \& E. Becerra, 2077 (USM, MO); 2650 m, 24 Octubre 2004, L. F. Mellado \& E. Becerra, 2097 (USM); 2650 m, 24 Octubre 2004, L. F. Mellado \& E. Becerra, 2103 (USM).

\section{Elaphoglossum puberulentum M. Kessler \& Mickel, Brittonia 58(2): 115. 2006}

Tipo: BOLIVIA. La Paz: Prov. Nor Yungas, Cantón Pacallo, Comunidad Chairo, Bajo Hornuni, subiendo $60 \mathrm{~m}$ altitudinales hacia el camino de la mina, $16^{\circ} 12^{\prime} \mathrm{S}, 67^{\circ} 53^{\prime} \mathrm{W}, 2000 \mathrm{~m}, 8 \mathrm{Oct}$ 2002, Beck 28386 (holótipo: NY, isótipo: LPB).

Rizoma cortamente repente, erguido, 10-18 mm de diámetro; escamas del rizoma ovado-lanceoladas, con el ápice crispado, marrón castaño doradas, enteras, 10-15 x 3-5 mm, las escamas más viejas sin el ápice; filopodio presente, 0,3-0,5 cm de largo, cubierto por las escamas del tallo, algo inconspicuo; hojas fasciculadas, 65-98 (130) x 4-8 cm; pecíolo $1 / 3-1 / 5$ del largo de la hoja estéril; escamas del pecíolo ovadas, enteras, $5 \mathrm{x}$ $3 \mathrm{~mm}$, marrón-castañas, opacas, esparcidas; lámina elíptica, raro obovada, cartácea, ápice largamente acuminado mayormente ausente, base largamente atenuado, borde con una comisura de $1 \mathrm{~mm}$ más oscura que la lámina (cartilaginosa); nervaduras conspicuas, $1-2 \mathrm{~mm}$ espaciadas, en un ángulo de $75-80^{\circ}$ con respecto a la costa, libres; hidatodos ausentes; escamas de la lámina ausentes, tricomidia estrellada esparcida, costa con apariencia glabra, pero con muy finas escamas lineares (tricomidia) dando le apariencia puberulenta; hoja fértil más corta del largo de la hoja estéril; pecíolo menos de 1/2 de la longitud de la hoja fértil; lámina fértil linear-obovadas, ápice largamente acuminado, base decurrentes, borde entero; escamas interesporangiales y sobre la costa ausentes.

Hierba, epífita, generalmente creciendo sobre los troncos de helechos arbóreos, rara terrestre; 1130-2770 m de altitud. Conocida desde el bosque pre-montano hasta bosque montano húmedo.

Observaciones: Elaphoglossum puberulentum (Sec. Elaphoglossum, Subsec. Tenuifolia), se encuentra relacionada morfológicamente con E. ambiguum (Mett. ex H. Christ) Alston, diferenciándose de ella por presentar finas escamas lineares (tricomidia) dando le apariencia puberulenta sobre la lámina, así como por sus escamas del peciolo ovadas, enteras, $5 \times 3 \mathrm{~mm}$, marrón-castańas, opacas.

Material examinado: PERÚ: Dep. Pasco. Prov. Oxapampa. Huancabamba: Sector Quebrada Yanachaga (P. N. Yanachaga-
Chemillén), 2250 m, 10-feb-2004, L. F. Mellado \& A. Monteagudo, 0276 (USM, MO, HOXA); 0314 (USM, HOXA); 0333 (USM); 0343 (USM, MO); 2070 m, 14-set-2004, L. Franco Mellado N., 1709 (USM, MO, HOXA, HUT); 2100 m, 16-set-2004, L. Franco Mellado N., 1751 (USM, MO, HOXA); 1758 (USM, MO); 2220 m, 17 Octubre 2004, L. Franco Mellado N., J. Perea, E. Becerra \& J. Mateo, 1959 (USM, MO, HOXA, HUT); 2260 m, 18-set-2004, L. F. Mellado \& E. Becerra, 1810 (USM, MO, HOXA); Pozuzo: Huampal, sector Pan de Azúcar, 1130 m, 11-nov-2004, L. Franco Mellado N., 2213 (USM, MO, HOXA); Villa Rica: Centro poblado: Palma Centro Bocaz. 1700 m, 12-ene-2005, L. Franco Mellado N., E. Ortiz V., J. Mateo \& R. Francis J., 2367 (USM, MO, HOXA); 2760 m, 12-ene-2005, L. Franco Mellado N., E. Ortiz V., J. Mateo \& R. Francis J., 2373 (USM, MO, HOXA, HUT); 2700-2770 m, 12-ene-; 2005, L. Franco Mellado N., E. Ortiz V., J. Mateo \& R. Francis J., 2398 (USM, MO, HOXA); 2461 (USM, MO); 1515 m, 14-ene-2005, L. Franco Mellado N., E. Ortiz V., J. Mateo \& R. Francis J., 2476 (USM, MO).

\section{Agradecimientos}

Al Fondo Christensen, que a través del Jardín Botánico de Missouri, auspició del proyecto para el estudio de la taxonomía y ecología del género Elaphoglossum en el Parque Nacional Yanachaga-Chemillén. Nuestros agradecimientos al personal de los Herbarios San Marcos (USM) y de la colección Herbario Selva central-HOXA del jardín Botánico de Missouri por las facilidades para la revisión de las muestras botánicas.

\section{Literatura citada}

Jørgensen P. M. \& S. León-Yánez, eds. 1999. Catalogue of the vascular plants of Ecuador. Monogr. Syst. Bot. Missouri Bot. Gard. 75: 1-1181.

Kessler M., \& J. T. Mickel, 2006. Nineteen new species of Elaphoglossum (Elaphoglossaceae, Pteridophyta) from Bolivia. Brittonia , 58 (2): 93-118.

León B. 2007[2006]. Lomariopsidaceae endémicas del Perú. En B. León, J. Roque, C. Ulloa Ulloa, N. Pitman, P.M. Jørgensen \& A. Cano (eds.). Libro Rojo de las Plantas endémicas del Perú. Rev. peru. biol. Número especial 13(2): 906s--910s

Mellado L.F. 2007. “Estudios Taxónomico y Ecológico del Género Elaphoglossum (Elaphoglossaceae) en el Parque Nacional Yanachaga-Chemillén,Oxapampa, Pasco" Tesis para optar Título Biólogo, Fac. Ciencias Biológicas, Universidad Nacional Mayor de San Marcos, Lima, Perú.

Mellado L. F., \& B. León, 2007. Nuevos registros y observaciones de algunas especies de Elaphoglossum (Elaphoglossaceae) del Perú. Rev. peru. biol., número especial 14 (1): 021 023.

Mickel J. T. 1991. Elaphoglossum. En R. M. Tryon, \& R. G. Stolze, Pteridophyta of Peru. Part IV 17. Dryopteridaceae. Fieldiana, Bot., n.s. 27: 111--270.

Mickel J. T., \& L. Atehortúa, 1980. Subdivision of the genus Elaphoglossum. American Fern J., 70 (2): 047-068.

Navarrete H. 2000. Pteridophyta. 31-49 pp. En R. Valencia, N. Pitman \& S. León Yánez, eds. Libro Rojo de las Plantas Endémicas del Ecuador. Quito, Ecuador.

Rojas-Alvarado A. F. 2002. New Species, New Combinations and new Distributions Species of Elaphoglossum (Lomariopsidaceae). Rev. Biol. Trop., 50 (3-4): 969-1006.

Smith, A.R., K.M. Pryer, E. Schuettpelz, P. Korall, H. Schneider \& P.G. Wolf. 2006. A classification of extant ferns. Taxon 55(3): 705--731. 


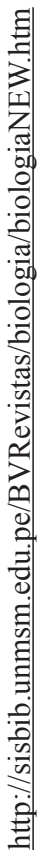

